\title{
The Use of Predictive Methods for the Design of New Explosive Molecules
}

\author{
A. Delpuech \\ Commissariat à l'Energie Atomique, Direction des Applications Militaires, France
}

This speech has two objectives :

- firstly, to show how the use of predictive methods is important during the search for new explosives,

- secondly, to illustrate with an exemple, the reliability of these methods.

Leaving aside considerations linked to the philosophy of individual organizations, the chances of a new molecule being successful involve the combination of at least two factors :

- the first is that it represents a significant advance in comparison with molecules being used at present. There is no doubt that the combination of the energy of HMX and the insensitivity of TATB makes for a major target and an ambitious objective,

- the second is that this new molecule achieves a wide field of application. This raises the factor of cost.

Thus with today's development budgets being more and more reduced, there is a clash between increasingly restrictive specifications :

- high energy performance,

- high safety (shock, heat),

- low cost.

And it is because of this that the need arises to make use of a molecular engineering process, a process based on the use of predictive methods with the double objectives of predicting, a priori and before synthesis, not only the explosive's characteristics (energy and safety), but also of evaluating development costs.

How is it possible to start "blind" on the search for a new molecule, given that the probability of obtaining a molecule which will have high energy performance (calculated as $80 \%$ of the energy of HMX) can perhaps be estimated as one chance in ten, a probability which becomes one chance in a million when the molecule has to combine the four requirements of high energy, low shock sensitivity, high thermal stability $\left(>300^{\circ} \mathrm{C}\right)$ and reasonable cost $(<100 \$ / \mathrm{Kg})$.

Another consideration must be discussed here.

The substantial advances required in comparison with molecules already in existence mean that those molecules able to respond to these ambitious objectives will belong to new families.

It is thus necessary to consider the use of new predictive methods which are not only empirical but also based on a knowledge of the relationship between the microscopic and the macroscopic. In other words, to develop a large number of tools which are at the same time theoretical or experimental, and which will allow the linking of the molecule's structural parameters with its explosive properties (energy, safety).

We have been engaged on this work at the DAM for close on twenty years. Today this allows us to synthesize new explosive molecules, designed on paper by use of predictive methods and responding to a specification which takes into account all the requirements : 
- safety requirements : shock sensitivity and thermal stability,

- energy performance : detonation pressure and velocity and above all delivered energy,

- cost

Predictive methods associated with each of these requirements have been developed in our laboratories, with the exception of density. Their use enables us to meet the specifications and leads to a proposed molecule.

This proposed molecule is analyzed by use of an expert system for computer-assisted synthesis. This system of course takes into account the chemical reactions involved in this type of synthesis; above all it combines all the expertise which we have acquired in this field since we began. While the use of this tool allows the research worker to analyse the most likely synthetic paths, it more importantly reveals the difficulties associated with the project, helping to plan for them, and thus to analyze the costs.

The complete process is illustrated in fig. 1 .

Before illustrating this with the results, I would like to make two comments :

- the first concerns the difficulty, according to the property being considered, of achieving a predictive method.

The evaluation of this difficulty is of course totally subjective. However it results from a consideration of the efforts which we have had to make to respond to the need. It is thus that it would appear to be more difficult to achieve a predictive tool giving values for detonation velocity and pressure at the CJ point than for density. Even more effort is therefore needed to determine the power supplied by the explosive as a function of time and expansion volume. These two properties relating to safety are in themselves much the most difficult to forecast.

- the second statement concerns the reliability of these methods. I am not going to detail here the methods which we use. At the reliability level, I would say that paradoxically, it is the density which poses us the most problems (in particular in the case of new molecules which do not belong to the traditional families). Since we know that this value is fundamental to a determination of the energy properties, this is a real problem.

In other cases the reliability is good. But this is wholly due to the fact that the methods which we use rely on the study of the true electronic or atomic structure of the molecules and make use of experiments in fundamental physics. It is completely different when we restrict ourselves to the use of empirical methods which indicate the changes in a property in terms of one parameter. Based on the results obtained for a large number of products, these empirical methods are not of any help in predicting the behaviour of a "truly" new product. It seems to me that an important point must be made here. It concerns the difficulty which still remains of evaluating not only the value of the power supplied by the explosive, but the way in which this power is supplied in time (an indispensable parameter for the optimization of the use of an explosive in terms of the munition volume). 
Let us return to one illustration of this step, by the presentation of the first molecule to be developed with the aid of this process.

This molecule had to respond to three objectives :

- a thermal stability requirement (decomposition temperature $>300^{\circ} \mathrm{C}$ ),

- a shock sensitivity requirement $(\mathrm{H} 50 \%(5 \mathrm{~kg})>60 \mathrm{~cm})$,

- an energy requirement : energy gain $/ \mathrm{TATB}=20 \%$.

While the double requirement energy-sensitivity is high, it is the thermal stability requirement which enormously increases the difficulty.

The design of the molecule made use of two molecular engineering steps :

First step : demonstration by quantum chemistry studies of the role played by the ANT unit in the insensitivity. Role confirmed by ultra-fast Raman spectrometry. Thermochemical study of this unit in addition permitted an estimation of its good energetic properties. On the other hand the thermostability of the compound was of the order of $240^{\circ} \mathrm{C}$.

Second step : grafting of this type of structure onto thermostable rings. The number of grafted ANT (1, 2 or 3 ) and the nature of the thermostable ring being optimized with respect to the energy properties.

The DANTNP molecule was constructed in this way. The molecule has been synthesized. An explosive composition of $97 \%$ DANTNP and $98 \%$ of the theoretical density has been developed.

The objective, the predicted values and the experimental results are shown in fig. 2 .

This molecule has of course limitations in application, chiefly its cost and the fact that its energy is delivered very progressively (from which arises the necessity of using large volumes). Its production nevertheless gives a perfect illustration of the contribution of predictive methods in such research.

The value of this contribution becomes even more telling when the changes which can be made to this molecule are considered in terms of different objectives, for example reduction of cost or optimization of the compromise between the different properties sought (sensitivity, thermostability and energy).

Much of the journey remains to be made.

But I firmly believe that only the use of reliable predicative methods will allow the constructive exploration of all four requirements and will enable us to find solutions as a function of the required specifications. Otherwise we shall be continuing to search for molecules "by going fishing". In that case we shall be spending a lot of money for very few results, apart from temporary and short-lived successes. I call temporary success the discovery of a molecule having one or at best two properties and which is then forgotten in a drawer after an ephemeral glory related to an abbreviated presentation of its properties. 


\section{CONCEPTION ET SYNTHESE DE NOUVELLES MOLECULES EXPLOSIVES}
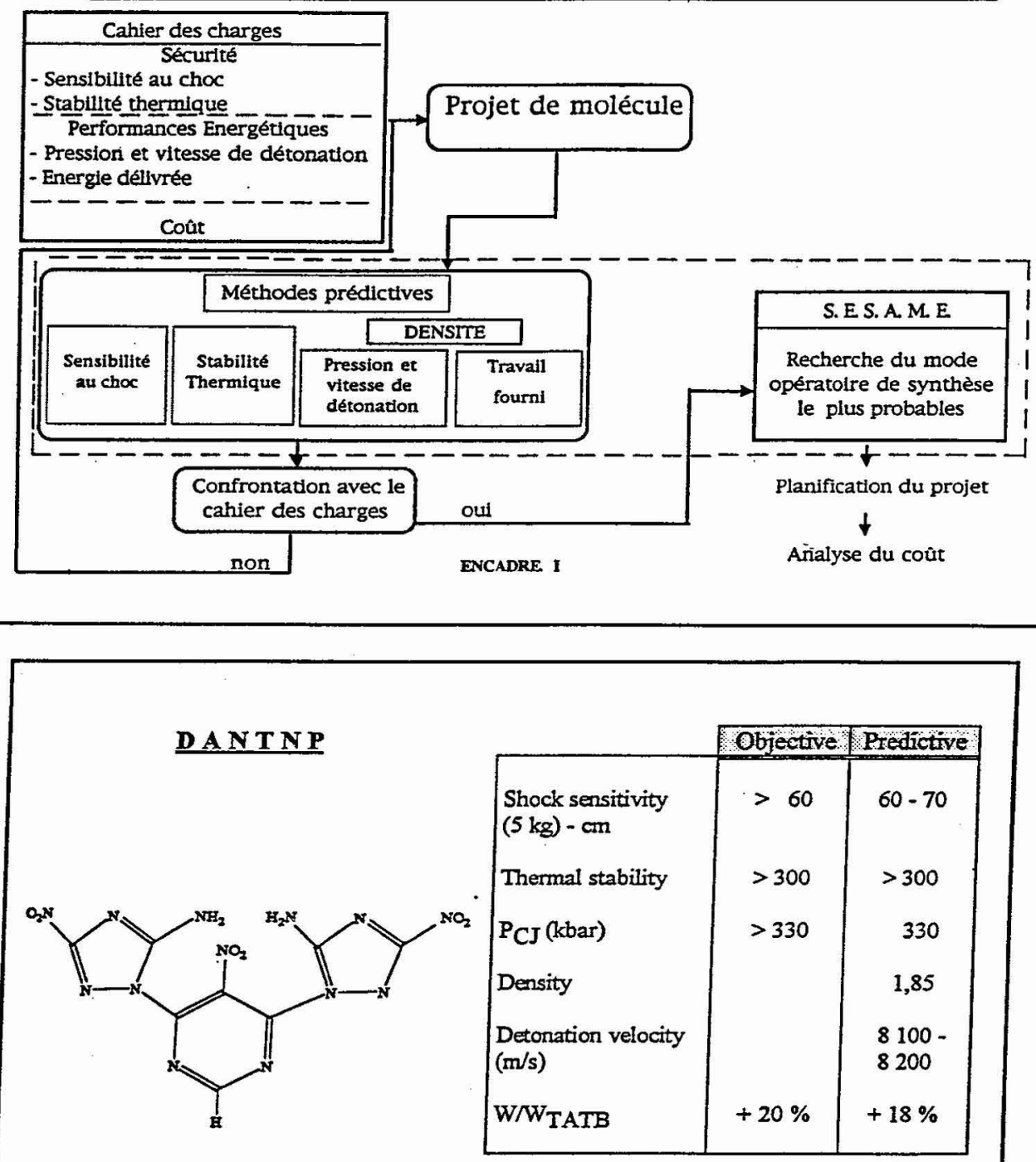

Experimental results

$\begin{array}{llll}\text { Density (XR) } & 1,865 & \text { Decomposition } \mathrm{T}^{\circ} \text { (DSC) } & 350^{\circ} \mathrm{C} \\ \text { Detonation velocity } & \mathbf{8 2 0 0 \mathrm { m } / \mathrm { s }} & \text { Deflagration } \mathrm{T}^{\circ} & 338^{\circ} \mathrm{C} \\ \mathrm{P}_{\mathrm{CJ}} & 327 \mathrm{Kbar} & \text { Shock sensitivity (powder) } & 70 \mathrm{~cm} \\ \text { W/W }_{\text {TATB }} & +15 \% & \text { Friction sensitivity } & >36 \mathrm{kgf}\end{array}$

- Fig. 2 - 